\title{
Differences in Flow-Gradient Patterns Between Severe Bicuspid Aortic Stenosis and Severe Tricuspid Aortic Stenosis
}

- Mechanistic Insight From Multimodal Imaging -

\author{
Darae Kim, MD, PhD; Chi Young Shim, MD, PhD; Young Jin Kim, MD, PhD; \\ Kyungsun Nam, MD; Geu-Ru Hong, MD, PhD; Seung Hyun Lee, MD, PhD; \\ Sak Lee, MD, PhD; Jong-Won Ha, MD, PhD
}

\begin{abstract}
Background: We investigated the flow-gradient pattern characteristics and associated factors in severe bicuspid aortic stenosis (AS) compared with severe tricuspid AS.

Methods and Results: A total of 252 patients with severe AS (115 bicuspid vs. 137 tricuspid) who underwent aortic valve (AV) replacement were retrospectively analyzed. Patients were classified into 4 groups according to stroke volume index and mean pressure gradient across the AV [normal-flow-high-gradient (NF-HG), low-flow-high-gradient, normal-flow-low-gradient, low-flow-lowgradient (LF-LG)]. In 89 patients who underwent cardiac computed tomography (CT), influential structural parameters of the left ventricular outflow tract (LVOT), AV and ascending aorta were assessed. Bicuspid AS was more likely to present a NF-HG pattern (83.5\% vs. $64.2 \%, P<0.001$ ), and significantly fewer presented a LF-LG pattern compared with tricuspid AS. In bicuspid AS, there was a significant mismatch between geometric orifice area (GOA) on CT planimetry and effective orifice area (EOA) calculated using the echocardiographic continuity equation. Bicuspid AS presented with a larger angle between the LVOT-AV and aorta. Multivariate analysis of bicuspid AS revealed that systemic arterial compliance $(\beta=-0.350, P=0.031)$ and the LVOT-AV-aorta angle $(\beta=-0.538$, $P=0.001)$, and stroke volume index $(\beta=0.409, P=0.008)$ were associated with a discrepancy between $G O A$ and EOA.
\end{abstract}

Conclusions: Flow-gradient patterns in bicuspid AS differ from those of tricuspid AS and are associated with the structural and functional characteristics of the aorta.

Key Words: Bicuspid aortic stenosis; Cardiac computed tomography; Echocardiography

A bicuspid aortic valve (BAV) is associated with early valve calcification, valvular tissue remodeling, and sclerosis. ${ }^{\mathbf{1 2}}$ Therefore, aortic stenosis (AS) is common with a BAV and typically occurs 1-2 decades earlier than it does with a tricuspid AV (TAV). ${ }^{\mathbf{3}, \mathbf{4}}$ Previous studies demonstrated that asymmetrical opening of the 2 leaflets results in altered geometry of the BAV, and that skewed forward flow accelerates leaflet calcification by increasing shear stress. ${ }^{\mathbf{5 6}}$ In addition, greater jet eccentricity in bicuspid AS can lead to variation between the geometric orifice area (GOA) and effective orifice area (EOA). ${ }^{7.8}$ An eccentric jet may result in increased pressure loss at the valve and ascending aorta and reduced pressure recovery distal to the orifice, which increases functional severity in bicuspid AS. ${ }^{9}$
The flow-gradient pattern in severe AS with preserved ejection fraction $(\mathrm{EF})$ has become an important parameter in the transcatheter AV replacement era because of its prognostic significance. ${ }^{10-12}$ Although many studies have presented the flow-gradient patterns in patients with severe AS, the patterns in patients with bicuspid AS have yet to be investigated. Because of greater jet eccentricity in bicuspid AS, different flow-gradient patterns in bicuspid AS compared with tricuspid AS are expected.

Thus, the aims of our study were to compare the flowgradient patterns in severe bicuspid AS with those in severe tricuspid AS, to compare the mismatch of GOA on cardiac computed tomography $(\mathrm{CT})$ planimetry and EOA calculated using the echocardiographic continuity equation in

Received August 2, 2019; revised manuscript received October 3, 2019; accepted October 22, 2019; J-STAGE Advance Publication released online November 30, 2019 Time for primary review: 20 days

Department of Medicine, Heart Vascular Stroke Institute, Samsung Medical Center, Sungkyunkwan University School of Medicine, Seoul (D.K.); Division of Cardiology, Severance Cardiovascular Hospital (C.Y.S., G.-R.H., J.-W.H.), Department of Radiology, Research Institute of Radiological Science, Severance Hospital (Y.J.K., K.N.), Department of Thoracic and Cardiovascular Surgery (S.H.L., S.L.), Yonsei University College of Medicine, Seoul, Republic of Korea

Mailing address: Chi Young Shim, MD, PhD, Associate Professor, Division of Cardiology, Severance Cardiovascular Hospital, Yonsei University College of Medicine, 50-1 Yonsei-Ro, Seodaemun-gu, Seoul, Korea, 120-752. E-mail: cysprs@yuhs.ac

ISSN-1346-9843 All rights are reserved to the Japanese Circulation Society. For permissions, please e-mail: cj@j-circ.or.jp 
bicuspid AS and tricuspid AS, and to explore the mechanisms of the different flow-gradient patterns in bicuspid AS using multimodal imaging.

\section{Methods}

\section{Study Population}

We identified consecutive patients who underwent surgical or transcatheter AV replacement at Severance Cardiovascular Hospital between January 2010 and December 2015. All patients were diagnosed with severe AS (AV area $<1.0 \mathrm{~cm}^{2}$ ) through comprehensive 2D and Doppler echocardiography according to the current guideline. ${ }^{13}$ Patients in our study underwent $\mathrm{AV}$ replacement in accordance with previous guidelines. ${ }^{14} \mathrm{~A}$ multidisciplinary heart team deemed patients eligible for transcatheter AV replacement if they were at intermediate or high risk for surgical AV replacement, defined as an estimated risk of 30-day surgical death. Patients with left ventricular EF $<50 \%$, other significant valve dysfunction (greater than moderate degree severity), prior open heart surgery, additional congenital abnormalities, dilated cardiomyopathy, hypertrophic cardiomyopathy, and atrial fibrillation were excluded. Finally, a total of 252 patients (128 men, mean age $69 \pm 10$ years) were included in this study. Among them, 115 patients had severe bicuspid AS and 137 had tricuspid AS. AV morphologies were confirmed by assessment of surgical specimens and/or multimodality imaging. Baseline clinical characteristics and laboratory results within 7 days preoperative were reviewed.

\section{Transthoracic Echocardiography}

All patients underwent comprehensive 2D transthoracic echocardiography within the month before surgical or transcatheter AV replacement. Standard measurements were performed according to the current guideline. ${ }^{13}$

BAV was diagnosed when only 2 cusps were unequivocally identified in systole and diastole in the short-axis view and BAV morphology was classified into the following 4 types according to the position and pattern of the raphe and cusps, as previously described:15,16 type $1: 1$ raphe with fusion of the left coronary and right coronary cusps; type 2: 1 raphe with fusion of the right coronary and non-coronary cusps; type 3: 1 raphe with fusion of the left coronary and non-coronary cusps; and type 0 : no raphe with 2 developed cusps. ${ }^{15-18}$

The left ventricular outflow tract (LVOT) diameter was measured during mid-systole $0.5-1 \mathrm{~cm}$ below the aortic annulus on the parasternal long-axis view. Stroke volume (SV) was calculated by multiplying the velocity time integral of systolic flow in the LVOT by the cross-sectional area of the LVOT calculated from the LVOT diameter. The ascending aorta was measured approximately $2 \mathrm{~cm}$ distal to the sinotubular junction. ${ }^{19}$

EOA was derived from the continuity equation. Highest peak velocity across the AV was measured from multiple windows, including apical, right parasternal, subcostal, and suprasternal notch views. Based on the mean pressure gradient (MPG) and SV index, patients were categorized into 4 groups: (1) low-flow-low-gradient (LF-LG) group: $\mathrm{SV}$ index $\leq 35 \mathrm{~mL} / \mathrm{m}^{2}$ and MPG $<40 \mathrm{mmHg}$; (2) low-flowhigh-gradient (LF-HG) group: SV index $\leq 35 \mathrm{~mL} / \mathrm{m}^{2}$ and MPG $\geq 40 \mathrm{mmHg}$; (3) normal-flow-low-gradient (NF-LG) group: SV index $>35 \mathrm{~mL} / \mathrm{m}^{2}$ and $\mathrm{MPG}<40 \mathrm{mmHg}$; (4) normal-flow-high-gradient (NF-HG) group: SV index
$>35 \mathrm{~mL} / \mathrm{m}^{2}$ and $\mathrm{MPG} \geq 40 \mathrm{mmHg}$. In addition, the normalflow-very high gradient group (NF-very HG group) was defined as $\mathrm{SV}$ index $>35 \mathrm{~mL} / \mathrm{m}^{2}$ and $\mathrm{MPG} \geq 50 \mathrm{mmHg}$.

\section{Hemodynamic Assessment}

Systolic arterial pressure was measured at the time of echocardiography using an arm cuff sphygmomanometer, and pulse pressure was derived as: systolic blood pressure (BP)diastolic BP. Total systemic arterial compliance (SAC) was calculated as previously described: SV index/pulse pressure. ${ }^{20}$ Mean arterial pressure was calculated as (systolic $\mathrm{BP}+2 \times$ diastolic $\mathrm{BP}) / 3$. Cardiac output was derived from Doppler measurement. ${ }^{21}$ Systemic vascular resistance was defined as $(80 \times$ mean arterial pressure)/cardiac output. Valvuloarterial impedance was measured as previously described: (systolic BP+MPG)/SV index. ${ }^{22}$

\section{Cardiac CT}

A total of 89 patients underwent cardiac CT within the month before surgical or transcatheter AV replacement at the physician's discretion as a means of preprocedural evaluation. All CT scans were performed with a dualsource CT scanner (SOMATOM Definition Flash; Siemens Healthcare, Forchheim, Germany). Scans were performed in retrospective ECG-gated data acquisition mode using the triple-phase injection method $(70 \mathrm{~mL}$ of iopamidol followed by $30 \mathrm{~mL}$ of $30 \%$ blended iopamidol with saline and $20 \mathrm{~mL}$ of saline at $5 \mathrm{~mL} / \mathrm{s}$ ).

Images were generated using iterative reconstruction (sinogram-affirmed iterative reconstruction). Image reconstruction was performed with a medium kernel (I36f), and the reconstruction slice thickness was $0.75 \mathrm{~mm}$ with $0.5-\mathrm{mm}$ increments. For all patients, 10 transverse data sets were reconstructed every $10 \%$ of the cardiac cycle. Image analysis was performed using 3D software (Aquarius iNtuition, Ver. 4.4.11, TeraRecon, San Mateo, CA, USA). For planimetry, the image volume was rotated into a plane perpendicular to the LVOT and aortic root. ${ }^{23}$ LVOT imaging involved orientation of a cross-sectional plane of the LVOT using 3 orthogonal planes from multiplanar reconstruction at or immediately under the lowest implantation base of the aortic cusp, and 2 orthogonal diameters (shortest, longest) were measured. ${ }^{24} \mathrm{GOA}$ was defined as the CT planimetry-derived AV area. Planimetry of the AV area was performed at the level of the aortic leaflet tips in the mid-systolic phase that provided the best visualization of the open aortic valve, usually at $20-30 \%$ of the R-R interval. Planimetry of the LVOT was performed immediately below the AV in the same phase used for measurement of the AV area. The angle between the LVOT-AV and aorta $\left({ }^{\circ}\right)$ was measured during the mid-systolic phase. Leaflet calcium was quantified by a standard Agatston methodology, with a threshold for calcium detection set at 130 Hounsfield units. 25

All CT analyses were independently performed by 2 radiologists blinded to clinical information, echocardiographic results, and CT analysis results of the other reader. When there was a disagreement between $\mathrm{CT}$ readers, the final decision was made through consensus.

\section{Statistical Analysis}

Categorical variables, expressed as percentages or frequencies, were compared between bicuspid and tricuspid AS groups using the $\chi^{2}$ test or Fisher's exact test. Continuous variables expressed as mean \pm standard deviation or median 


\begin{tabular}{|lcrr|}
\hline Table 1. Patients' Baseline Characteristics & & & \\
& $\begin{array}{c}\text { Bicuspid } \\
(\mathbf{n}=\mathbf{1 1 5})\end{array}$ & $\begin{array}{c}\text { Tricuspid } \\
(\mathbf{n}=\mathbf{1 3 7})\end{array}$ & P value \\
Age, years & $63.3 \pm 8.8$ & $73.4 \pm 7.3$ & $<0.001$ \\
Male sex, $\mathrm{n}(\%)$ & $58(55)$ & $67(48)$ & 0.302 \\
Height, cm & $161.8 \pm 9.6$ & $159.3 \pm 8.4$ & 0.033 \\
Weight, kg & $63.7 \pm 11.0$ & $61.1 \pm 10.6$ & 0.061 \\
Body mass index, kg/m² & $24.6 \pm 4.2$ & $24.7 \pm 3.2$ & 0.223 \\
Systolic BP, mmHg & $124 \pm 16$ & $125 \pm 16$ & 0.443 \\
Diastolic BP, mmHg & $76 \pm 12$ & $71 \pm 12$ & $<0.001$ \\
Heart rate, beats/min & $66 \pm 10$ & $66 \pm 12$ & 0.484 \\
Comorbidities & & & \\
Hypertension, $\mathrm{n}(\%)$ & $63(60)$ & $107(77)$ & $<0.001$ \\
Diabetic mellitus, $\mathrm{n}(\%)$ & $25(24)$ & $43(63)$ & 0.252 \\
CKD, $\mathrm{n}(\%)$ & $5(4)$ & $18(13)$ & 0.020 \\
Dyslipidemia, $\mathrm{n}(\%)$ & $29(25)$ & $38(28)$ & 0.537 \\
Log NT-proBNP, pg/mL & $2.89 \pm 0.52$ & $3.02 \pm 0.76$ & 0.534 \\
\hline
\end{tabular}

$\mathrm{BP}$, blood pressure; CKD, chronic kidney disease; NT-proBNP, N-terminal prohormone of B-type natriuretic peptide.

\begin{tabular}{|c|c|c|c|}
\hline & $\begin{array}{c}\text { Bicuspid } \\
(n=115)\end{array}$ & $\begin{array}{c}\text { Tricuspid } \\
(\mathrm{n}=137)\end{array}$ & $P$ value \\
\hline \multicolumn{4}{|l|}{ Ventricular characteristics } \\
\hline LVEDD, $\mathrm{mm}$ & $48.3 \pm 4.2$ & $48.8 \pm 6.1$ & 0.556 \\
\hline LVESD, mm & $30.8 \pm 4.3$ & $31.8 \pm 6.6$ & 0.202 \\
\hline LV mass index, $\mathrm{g} / \mathrm{m}^{2}$ & $137.4 \pm 31.2$ & $138.2 \pm 37.2$ & 0.945 \\
\hline LV ejection fraction, \% & $68.3 \pm 7.2$ & $66.3 \pm 11.1$ & 0.132 \\
\hline $\mathrm{LA}$ volume index, $\mathrm{mL} / \mathrm{m}^{2}$ & $34.2 \pm 10.5$ & $45.0 \pm 22.8$ & $<0.001$ \\
\hline E/ septal e' & $15.6 \pm 5.1$ & $20.0 \pm 8.1$ & $<0.001$ \\
\hline $\mathrm{RVSP}, \mathrm{mmHg}$ & $28.5 \pm 6.7$ & $32.3 \pm 11.4$ & $<0.001$ \\
\hline \multicolumn{4}{|l|}{ Flow-gradient characteristics } \\
\hline Peak pressure gradient, $\mathrm{mmHg}$ & $95.7 \pm 25.4$ & $86.5 \pm 35.6$ & $<0.001$ \\
\hline MPG, $\mathrm{mmHg}$ & $61.2 \pm 17.2$ & $51.8 \pm 17.8$ & $<0.001$ \\
\hline Peak transaortic velocity, $\mathrm{m} / \mathrm{s}$ & $4.9 \pm 0.6$ & $4.5 \pm 0.7$ & $<0.001$ \\
\hline $\mathrm{AV}$ area by $\mathrm{CE}, \mathrm{cm}^{2}$ & $0.67 \pm 0.15$ & $0.77 \pm 0.20$ & $<0.001$ \\
\hline Stroke volume index, $\mathrm{mL} / \mathrm{m}^{2}$ & $45.5 \pm 9.1$ & $47.7 \pm 11.5$ & 0.112 \\
\hline LVOT diameter, mm & $22.1 \pm 1.5$ & $20.9 \pm 1.8$ & $<0.001$ \\
\hline LVOT time velocity integral, $\mathrm{cm}$ & $21.2 \pm 3.7$ & $22.7 \pm 5.1$ & 0.014 \\
\hline \multicolumn{4}{|l|}{ Flow-gradient classification } \\
\hline NF-HG, n (\%) & $97(84)$ & $88(64)$ & $<0.001$ \\
\hline NF-LG, n (\%) & $7(8)$ & $28(20)$ & 0.001 \\
\hline LF-HG, n (\%) & $10(9)$ & $12(9)$ & 0.487 \\
\hline LF-LG, n (\%) & $1(1)$ & $9(7)$ & 0.019 \\
\hline \multicolumn{4}{|l|}{ Vascular characteristics } \\
\hline Ascending aorta diameter, $\mathrm{mm}$ & $41.7 \pm 6.0$ & $36.6 \pm 4.9$ & $<0.001$ \\
\hline $\mathrm{SAC}, \mathrm{mL} \cdot \mathrm{m}^{-2} \cdot \mathrm{mmHg}^{-1}$ & $1.03 \pm 0.30$ & $0.93 \pm 0.30$ & 0.022 \\
\hline SVR, dyne $\cdot \mathrm{s} \cdot \mathrm{cm}^{-5}$ & $1,552 \pm 371$ & $1,518 \pm 8,493$ & 0.551 \\
\hline \multicolumn{4}{|l|}{ Valvuloarterial impedance } \\
\hline $\mathrm{Zva}, \mathrm{mmHg} / \mathrm{mL} / \mathrm{m}^{2}$ & $4.19 \pm 0.93$ & $3.96 \pm 1.26$ & 0.128 \\
\hline \multicolumn{4}{|l|}{ BAV morphology } \\
\hline Type 1 (RCC+LCC), n (\%) & $68(59)$ & - & \\
\hline Type 2 (RCC+NCC), n (\%) & $31(27)$ & - & \\
\hline Type 3 (LCC+NCC), n (\%) & $3(3)$ & - & \\
\hline Type 0 (No raphe), n (\%) & $13(11)$ & - & \\
\hline
\end{tabular}

$\mathrm{AV}$, aortic valve; $\mathrm{BSA}$, body surface area; $\mathrm{CE}$, continuity equation; $\mathrm{EDD}$, end-diastolic dimension; $\mathrm{ESD}$, endsystolic dimension; LA, left atrium; LV, left ventricle; LVOT, left ventricular outflow tract; MPG, mean pressure gradient; RVSP, right ventricular systolic pressure; SAC, systemic arterial compliance; SVR, systemic vascular resistance; Zva, valvuloarterial impendance. 


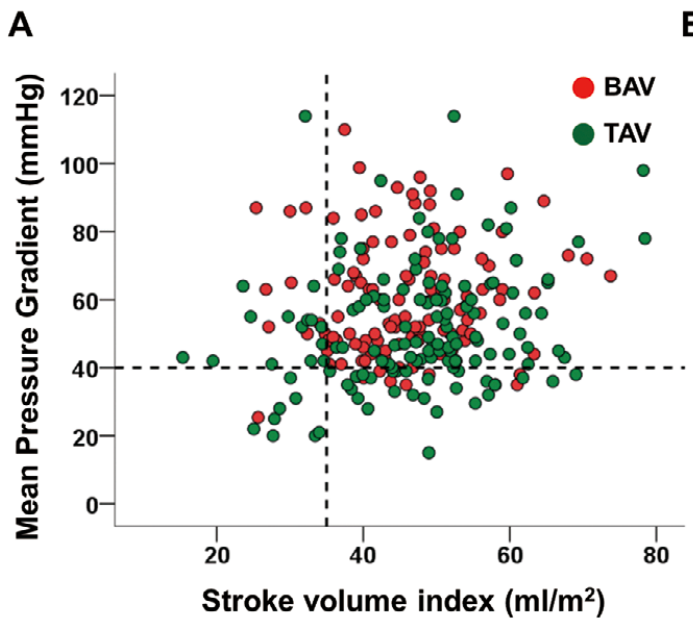

B

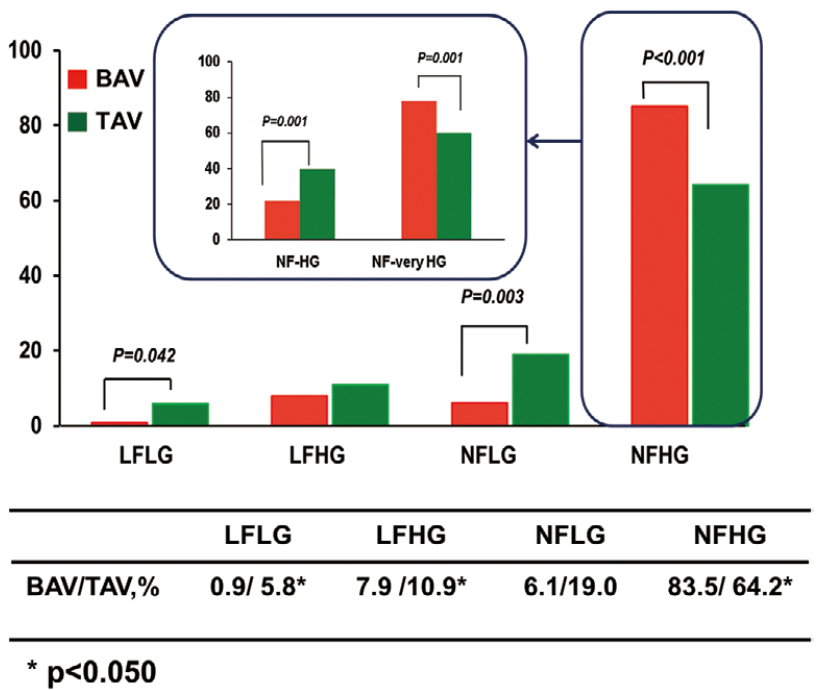

Figure 1. Flow-gradient patterns according to mean pressure gradient and stroke volume index in severe bicuspid AS and tricuspid AS patients. (A) Distribution according to stroke volume index and mean pressure gradient. (B) Prevalence of flow-gradient patterns. AS, aortic stenosis; BAV, bicuspid aortic valve; TAV, tricuspid aortic valve.

with range were compared between groups using Student's t-test. In the subgroup analysis, Spearman's simple correlation analyses were performed to determine parameters associated with the ratio of AV area (EOA/GOA) in bicuspid severe AS patients. Multiple linear regression analysis was performed to identify independent covariates of mismatch between EOA and GOA in bicuspid AS. Parameters that were significantly correlated from simple correlation analysis were included in the multivariate model. Age and ascending aortic diameter were included on the basis of our own hypothesis. Statistical analyses were performed using SPSS statistical package version 22.0 (SPSS Inc., Chicago, IL, USA).

\section{Results}

\section{Baseline Characteristics}

Baseline characteristics of the severe bicuspid AS and tricuspid AS patients are described in Table 1. Patients with bicuspid AS were significantly younger and had significantly fewer comorbidities, such as hypertension and chronic kidney disease, when compared with tricuspid AS patients. Average diastolic BP in patients with bicuspid AS was higher than that in tricuspid AS, probably because of the younger age and fewer comorbidities. However, mean log N-terminal-pro B-type natriuretic peptide levels were comparable between the 2 groups.

\section{Echocardiographic Characteristics and Flow-Gradient Patterns} A comparison of the echocardiographic variables is presented in Table 2. LV chamber size and LV mass index did not differ between the 2 groups, but the LA volume index was significantly smaller in the severe bicuspid AS group. Estimated LV filling pressure, reflected by E/septal e' and RVSP, was significantly lower in bicuspid AS. Peak pressure gradient, MPG and peak transaortic velocity were significantly higher in bicuspid AS, while EOA calculated using the continuity equation was significantly smaller in bicuspid AS compared with tricuspid AS. LVOT diameter and ascending aortic diameter were significantly larger in bicuspid AS. Regarding vascular load, SAC was significantly higher in bicuspid AS, while systemic vascular resistance and valvuloarterial impedance did not differ between groups. Among bicuspid AS patients, type 1 was the most prevalent phenotype.

Figure 1 describes the flow-gradient patterns according to MPG and the SV index in severe bicuspid and tricuspid AS patients. Bicuspid AS patients were more likely to present a NF-HG pattern ( $83.5 \%$ vs. $64.2 \%, \mathrm{P}<0.001)$, and less likely to present a LF-LG pattern $(0.9 \%$ vs. $5.8 \%, \mathrm{P}=0.042)$, when compared with the tricuspid AS group. Significantly more severe bicuspid AS patients $(78.1 \%$ vs. $60.2 \%, \mathrm{P}=0.010)$ presented with a very $\mathrm{HG}$ compared with tricuspid AS patients (Figure 1B).

In the bicuspid subgroup, patients presenting a NF-very HG pattern had significantly smaller EOA compared with those with the NF-HG pattern, while GOA did not differ between the 2 groups (Supplementary Tables 1,2). Also, bicuspid AS patients with NF-very HG had a significantly larger LVOT-AV-aorta angle and higher SAC when compared with those with the NF-HG pattern.

\section{EOA vs. GOA in Bicuspid and Tricuspid AS Patients}

Table 3 shows the valvular and vascular characteristics of 89 patients who underwent both echocardiography and cardiac CT. Although the average of EOA calculated via echo-Doppler was significantly smaller in bicuspid AS, the average GOA on CT planimetry was significantly larger in patients with bicuspid AS compared with tricuspid AS. In addition, the ascending aortic diameter and LVOT-AVaorta angle were significantly larger in severe bicuspid AS patients. Therefore, the mismatch between EOA and GOA was more significant in severe bicuspid AS compared with tricuspid AS. The difference in AV area (GOA-EOA) was 


\begin{tabular}{|c|c|c|c|}
\hline & $\begin{array}{l}\text { Bicuspid } \\
(n=33)\end{array}$ & $\begin{array}{c}\text { Tricuspid } \\
(n=56)\end{array}$ & $P$ value \\
\hline \multicolumn{4}{|l|}{ Echocardiography } \\
\hline $\mathrm{AV}$ area $(\mathrm{EOA})$ by $\mathrm{CE}, \mathrm{cm}^{2}$ & $0.67 \pm 0.15$ & $0.76 \pm 0.18$ & $<0.0001$ \\
\hline Indexed $A V$ area $(E O A)$ by $C E, \mathrm{~cm}^{2} / \mathrm{m}^{2}$ & $0.40 \pm 0.09$ & $0.47 \pm 0.11$ & $<0.0001$ \\
\hline LVOT diameter, mm & $22.0 \pm 1.6$ & $20.9 \pm 1.8$ & $<0.0001$ \\
\hline LVOT area, $\mathrm{cm}^{2}$ & $3.84 \pm 0.54$ & $3.47 \pm 0.60$ & $<0.001$ \\
\hline Ascending aorta diameter, $\mathrm{mm}$ & $41.6 \pm 5.9$ & $36.5 \pm 4.7$ & $<0.0001$ \\
\hline \multicolumn{4}{|l|}{ Cardiac CT } \\
\hline $\mathrm{AV}$ area $(\mathrm{GOA})$ by planimetry, $\mathrm{cm}^{2}$ & $0.81 \pm 0.17$ & $0.72 \pm 0.24$ & 0.034 \\
\hline LVOT diameter (short), mm & $21.9 \pm 2.3$ & $21.4 \pm 2.45$ & 0.498 \\
\hline LVOT diameter (long), mm & $27.6 \pm 3.0$ & $28.0 \pm 2.62$ & 0.267 \\
\hline LVOT area, $\mathrm{cm}^{2}$ & $4.79 \pm 0.98$ & $4.73 \pm 0.93$ & 0.772 \\
\hline Annulus diameter, mm & $23.5 \pm 2.2$ & $23.6 \pm 1.9$ & 0.883 \\
\hline Ascending aorta diameter, $\mathrm{mm}$ & $42.5 \pm 7.1$ & $37.5 \pm 4.5$ & $<0.001$ \\
\hline LVOT-AV-aorta angle, ${ }^{\circ}$ & $16.3 \pm 7.7$ & $11.3 \pm 4.1$ & 0.002 \\
\hline $\mathrm{AV}$ calcium score, $\mathrm{AU}$ & $2,732 \pm 1,727$ & $3,360 \pm 1,634$ & 0.009 \\
\hline \multicolumn{4}{|l|}{ Comparison of LVOT and AV area } \\
\hline Difference of $\mathrm{AV}$ area (GOA-EOA), $\mathrm{cm}^{2}$ & $0.15 \pm 0.17$ & $-0.03 \pm 0.22$ & $<0.001$ \\
\hline Ratio of $A V$ area $(E O A / G O A)$ & $0.82 \pm 0.18$ & $1.13 \pm 0.40$ & $<0.001$ \\
\hline Ratio of LVOT area echo/LVOT area cт & $0.80 \pm 0.14$ & $0.79 \pm 0.12$ & 0.708 \\
\hline
\end{tabular}

$\mathrm{AU}$, Agatston unit; EOA, effective orifice area; GOA, geometric orifice area. Other abbreviations as in Table 2.

A

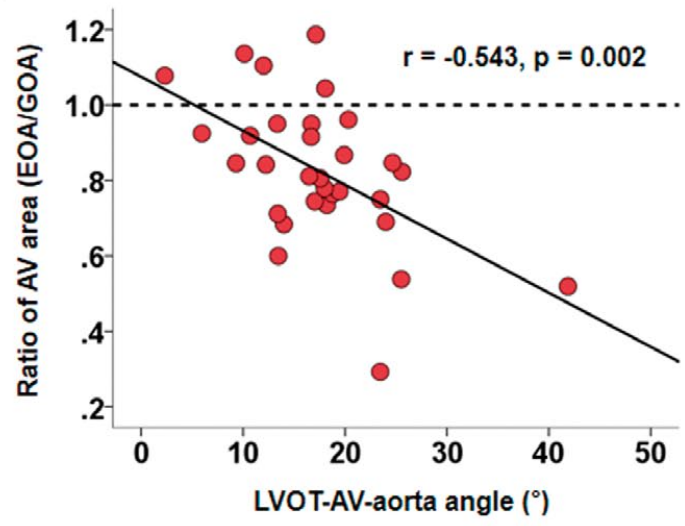

B

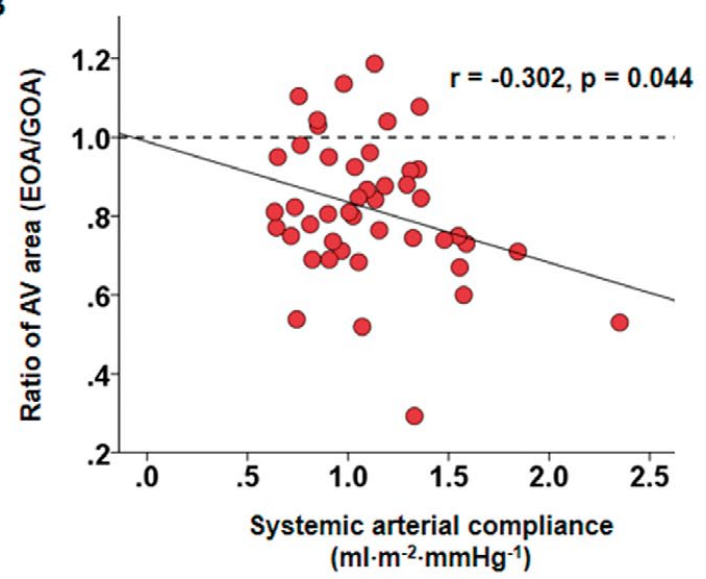

Figure 2. Simple correlations analysis. (A) Association of the ratio of $A V$ area (EOA/GOA) with the LVOT-AV-aorta angle shows a significant negative correlation. (B) Association of the ratio of $A V$ area (EOA/GOA) with systemic arterial compliance shows a significant negative correlation. AV, aortic valve; EOA, effective orifice area; GOA, geometric orifice area; LVOT, left ventricular outflow tract.

significantly larger and the ratio of AV area (EOA/GOA) was significantly lower in the severe bicuspid AS group when compared with the tricuspid AS group. AV leaflet calcium scores were significantly higher in the tricuspid AS group. The ratio of LVOT area echo/LVOT area CT was similar between the 2 groups.

Figure 2 shows the simple correlations analysis of the LVOT-AV-aorta angle and SAC to the ratio of AV area (EOA/GOA) in the bicuspid AS group. Both the LVOT$\mathrm{AV}$-aorta angle $(\mathrm{r}=-0.543, \mathrm{P}=0.002)$ and $\mathrm{SAC}(\mathrm{r}=-0.302$,
$\mathrm{P}=0.044)$ showed significant negative correlations to the ratio of $\mathrm{AV}$ area (EOA/GOA). The SV index revealed significant positive correlations with the ratio of $\mathrm{AV}$ area $(\mathrm{EOA} / \mathrm{GOA})(\mathrm{r}=0.443, \mathrm{P}<0.001)$. Multivariate analysis revealed that the SV index, SAC, and LVOT-AV-aorta angle were independently associated with the mismatch between EOA and GOA in severe bicuspid AS (Table 4).

Figure 3 shows representative cases. Severe bicuspid AS patients with prominent EOA-GOA mismatch presented with a larger LVOT-AV-aorta angle (Figure 3A). Severe 


\begin{tabular}{|c|c|c|c|}
\hline & $\beta$ & $\mathbf{T}$ & $\mathbf{p}$ \\
\hline \multicolumn{4}{|c|}{ Ratio of $A V$ area $(E O A / G O A)\left(R^{2}=0.597\right)$} \\
\hline Age & -0.117 & -0.679 & 0.503 \\
\hline Stroke volume index, $\mathrm{mL} / \mathrm{m}^{2}$ & 0.409 & 2.820 & 0.008 \\
\hline $\mathrm{SAC}, \mathrm{mL} \cdot \mathrm{m}^{-2} \cdot \mathrm{mmHg}^{-1}$ & -0.350 & -2.359 & 0.031 \\
\hline Ascending aorta diameter, $\mathrm{mm}$ & 0.025 & 0.175 & 0.749 \\
\hline LVOT-AV-aorta angle, $^{\circ}$ & -0.538 & -3.694 & 0.001 \\
\hline
\end{tabular}

AS, aortic stenosis. Other abbreviations as in Tables 2,3.

\section{A LVOT-AV-aorta angle}

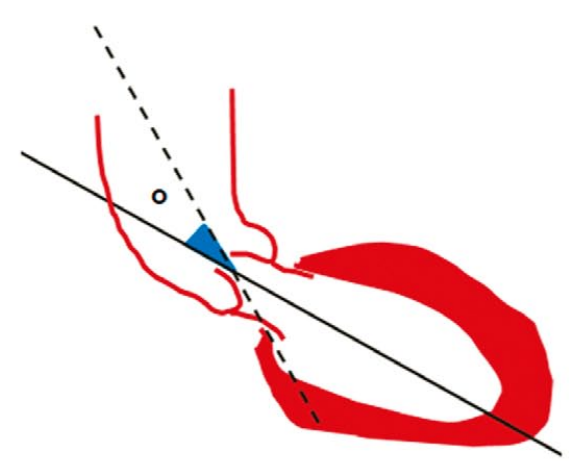

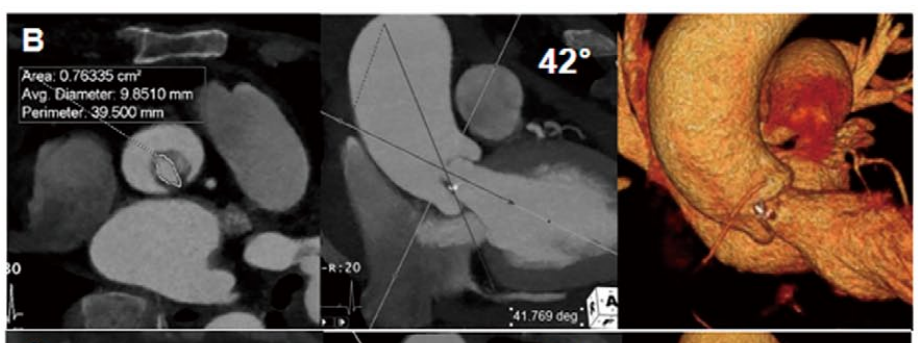
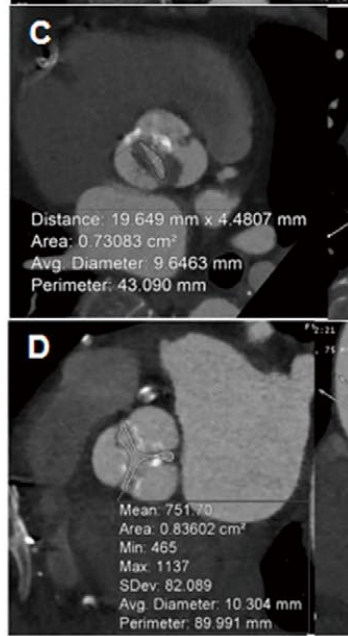
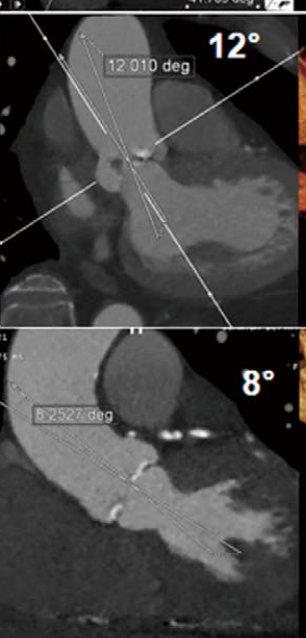

Figure 3. Representative cases. (A) LVOT-AV-aorta angle measured from cardiac CT. (B) Bicuspid AS with higher discrepancy between EOA and GOA (ratio: 0.52) shows larger LVOT-AV-aorta angle $\left(42^{\circ}\right)$. (C) Bicuspid AS with less discrepant EOA and GOA (ratio: 1.10) shows smaller LVOT-AV-aorta angle (12'). (D) Tricuspid AS without significant discrepancy between EOA and GOA area (ratio: 1.04) has LVOT-AV-aorta angle of $8^{\circ}$. Abbreviations as in Figure 2.

bicuspid AS patients with a less significant mismatch between EOA and GOA had a smaller LVOT-AV-aorta angle (Figure 3B). Severe tricuspid AS led to a less significant mismatch between EOA and GOA and a smaller LVOT-AV-aorta angle (Figure 3C).

\section{Discussion}

The principle findings of the present study were: (1) significantly more of the severe bicuspid AS patients presented with a NF-HG pattern compared with severe tricuspid AS patients; (2) the paradoxical LF-LG pattern was significantly less prevalent in bicuspid AS than in tricuspid AS; (3) the mismatch between EOA and GOA, reflected by the ratio of AV area calculated using the con- tinuity equation to $\mathrm{AV}$ area on CT planimetry, was more significant in bicuspid AS compared with tricuspid AS; and (4) the LVOT-AV-aorta angle and SAC were independently associated with EOA and GOA mismatch in bicuspid AS. The results of multimodal imaging suggested that bicuspid AS has different flow-gradient patterns from tricuspid AS related to the structural and functional characteristics of the aorta.

The flow-gradient pattern is an important issue in AS, not only from a diagnostic point of view, but also because flow is closely related to prognosis. ${ }^{26}$ However, few studies have explored the flow-gradient patterns of bicuspid AS. In this study, the prevalence of the NF-HG pattern was significantly higher in bicuspid AS patients, and a very HG pattern was significantly more prevalent in bicuspid AS 
when compared with tricuspid AS. Significantly fewer bicuspid AS patients presented with the paradoxical LF-LG pattern compared with tricuspid AS patients. In our cohort, however, the total prevalence of the paradoxical LF-LG pattern was 2.8\% (7 of 252 patients), which is much lower than in previous studies. ${ }^{27,28}$ This is because we excluded patients with other significant valvular heart disease and atrial fibrillation (important factors in paradoxical LF-LG) in order to increase the accuracy of the AV hemodynamic measurements with minimal confounding variables and limitations. Nonetheless, we showed that significantly fewer bicuspid AS patients had the LF-LG pattern when compared with tricuspid AS patients ( $1 \%$ vs. $7 \%, \mathrm{P}=0.019)$. This finding is consistent with the previous study of Clavel et al, ${ }^{29}$ who statistically compared the weight of the AV between severe AS patients with a paradoxical LF-LG pattern and a NF-HG pattern and demonstrated a significantly higher proportion of bicuspid AS patients in the NF-HG group ( $42 \%$ vs. $15 \%, \mathrm{P}=0.003)$ than in the paradoxical LF-LG group. Higher mean gradient in bicuspid AS, compared with tricuspid AS, may be explained by better tolerance of AS symptoms. Better-preserved diastolic function and arterial compliance in bicuspid AS patients of relatively younger age may play a role, suggesting AS is not just a disease confined to the AV, but rather a disease continuum involving the left ventricle, AV, and aorta.

Although valve calcification is known to occur earlier in bicuspid AV, AV calcium scores were significantly lower in bicuspid AS than in tricuspid AS, possibly because of the older age of the tricuspid AS patients. The AV calcium score showed significant correlation with MPG (Supplementary Figure A), although in the bicuspid subgroup analysis, such correlation failed to remain significant, whereas in the tricuspid subgroup, the correlation appeared to be even stronger (Supplementary Figure B,C).

The prognostic implications of the different flow-gradient patterns in bicuspid and tricuspid AS patients were beyond the scope of this study and will require further investigation.

Mismatch between the EOA and GOA was more significant in bicuspid AS when compared with tricuspid AS. Previous study using a fluid-structure interaction model showed that prediction of maximum EOA according to valve anatomy (bicuspid vs. tricuspid) revealed that BAV was associated with a $49 \%$ decrease in EOA on average compared with TAV..$^{30}$ This finding is consistent with our results, in which severe bicuspid AS had significantly higher peak transvalvular velocity, higher MPG, and larger GOA on CT planimetry, while the EOA calculated using the echocardiographic continuity equation was significantly smaller in bicuspid AS when compared with tricuspid AS. We also examined the ratio of LVOT area echo/LVOT area CT to assess if underestimation of LVOT area in patients with bicuspid AS could explain the mismatch between EOA and GOA in the bicuspid group, compared with tricuspid AS. The mean value of LVOT area echo/LVOT area CT was $<1$ in both groups, reflecting underestimation of LVOT area by echocardiography. However, the mean value was similar in both groups, suggesting that the degree of underestimation of the LVOT area was similar in both groups.

To determine the independent factors for mismatch between EOA and GOA, we measured the LVOT-AVaorta angle using $\mathrm{CT}$ to indirectly measure eccentric jet. In the multivariate analysis, the LVOT-AV-aorta angle was independently associated with mismatch between EOA and GOA. Our finding is similar to that of previous study using an AS computational flow dynamic model, which showed that with constant valve anatomic area, eccentric jet was associated with increased jet velocity and thus resulted in reduced EOA. ${ }^{31}$ Our result also suggested that eccentric jet results in a mismatch between EOA and GOA. SV index and SAC were also independently associated with mismatch between EOA and GOA. Positive correlation between the SV index and EOA/GOA ratio is not surprising considering the same components are used to calculate the SV index and EOA. The independent negative correlation between SAC and a mismatch between EOA and GOA is notable. Consequently, our finding suggested that the flow-gradient patterns in bicuspid AS are not limited to the AV and left ventricle, and that characteristics of the aorta may play an important role. Ultimately, multimodality imaging is needed to better understand the hemodynamics of AS. Phase-contrast magnetic resonance imaging (MRI) provides complementary information by enabling direct investigation of time-resolved velocities and energy dissipation. ${ }^{32} \mathrm{~A}$ previous study by Binter et a ${ }^{33}$ reported higher energy loss with bicuspid AV caused by eccentric jets and dilated aortic geometry using turbulent kinetic energy assessed by 4D flow MRI, which could not be assessed by the current echocardiographic parameters. Further longitudinal studies with multimodality imaging are needed to determine the clinical implications and prognostic role of the flow-gradient patterns in bicuspid AS patients.

\section{Study Limitations}

First, this study had a cross-sectional design and was conducted at a single center. Our study has the intrinsic limitations of retrospective design: longitudinal follow-up data were not included in this study. However, comprehensive hemodynamic and flow-gradient pattern data were compared between bicuspid and tricuspid AS patients. Echocardiography Doppler measurements and CT parameters successfully quantified jet eccentricity and mismatch between the GOA and EOA of the AV in a relatively large number of bicuspid AV patients compared with previous studies. Further studies with longitudinal data are needed to determine the prognostic implications of pressure gradient patterns in bicuspid AS patients. Second, because we excluded patients with atrial fibrillation and other significant valvular heart disease for the sake of precise measurement of AV hemodynamics, fewer patients with the paradoxical LF-LG pattern were enrolled compared with previous studies. Further studies that include atrial fibrillation patients are needed to evaluate the real-world prevalence of the LF-LG and NF-LG patterns in bicuspid AV patients. Third, the LVOT-AV-aorta angle measured on CT was used to indirectly represent jet eccentricity; however, more advanced imaging techniques such as 4D flow MRI may better demonstrate eccentric and helical jet types of aortic flow across the AV. Furthermore, advanced echocardiographic techniques, such as 3D echocardiography, would enhance the accuracy of LVOT and hemodynamic measurements. We did not measure other parameters, such as maximal and minimal valve orifice diameters, that may reflect jet eccentricity.

In conclusion, flow-gradient patterns appear to differ between bicuspid and tricuspid AS patients. Severe bicuspid AS mostly presents with a NF-HG pattern, especially a NF-very HG pattern, compared with severe tricuspid AS, while the paradoxical LF-LG pattern was significantly 
less prevalent in bicuspid AS. The LVOT-AV-aorta angle, SAC, and SV index were independently associated with a mismatch between GOA and EOA in bicuspid AS. The structural and functional characteristics of bicuspid AS appear to be attributable to the distinct flow-gradient patterns.

\section{Acknowledgments / Conflict of Interest / Funding}

None.

\section{References}

1. Lewin MB, Otto CM. The bicuspid aortic valve: Adverse outcomes from infancy to old age. Circulation 2005; 111: 832-834.

2. Ward C. Clinical significance of the bicuspid aortic valve. Heart 2000; 83: 81-85.

3. Hope MD, Urbania TH, Yu JP, Chitsaz S, Tseng E. Incidental aortic valve calcification on CT scans: Significance for bicuspid and tricuspid valve disease. Acad Radiol 2012; 19: $542-547$.

4. Pomerance A. Pathogenesis of aortic stenosis and its relation to age. Br Heart J 1972; 34: 569-574.

5. Robicsek F, Thubrikar MJ, Cook JW, Fowler B. The congenitally bicuspid aortic valve: How does it function? Why does it fail? Ann Thorac Surg 2004; 77: 177-185.

6. den Reijer PM, Sallee D 3rd, van der Velden P, Zaaijer ER, Parks WJ, Ramamurthy S, et al. Hemodynamic predictors of aortic dilatation in bicuspid aortic valve by velocity-encoded cardiovascular magnetic resonance. J Cardiovasc Magn Reson 2010; 12: 4.

7. Donal E, Novaro GM, Deserrano D, Popovic ZB, Greenberg NL, Richards KE, et al. Planimetric assessment of anatomic valve area overestimates effective orifice area in bicuspid aortic stenosis. J Am Soc Echocardiogr 2005; 18: 1392-1398.

8. Abbas AE, Franey LM, Goldstein J, Lester S. Aortic valve stenosis: To the gradient and beyond: The mismatch between area and gradient severity. J Interv Cardiol 2013; 26: 183-194.

9. Richards KE, Deserranno D, Donal E, Greenberg NL, Thomas JD, Garcia MJ. Influence of structural geometry on the severity of bicuspid aortic stenosis. Am J Physiol Heart Circ Physiol 2004; 287: H1410-H1416.

10. Hachicha Z, Dumesnil JG, Bogaty P, Pibarot P. Paradoxical low-flow, low-gradient severe aortic stenosis despite preserved ejection fraction is associated with higher afterload and reduced survival. Circulation 2007; 115: 2856-2864.

11. Dumesnil JG, Pibarot P, Carabello B. Paradoxical low flow and/ or low gradient severe aortic stenosis despite preserved left ventricular ejection fraction: Implications for diagnosis and treatment. Eur Heart J 2010; 31: 281 - 289.

12. Mohty D, Magne J, Deltreuil M, Aboyans V, Echahidi N, Cassat $\mathrm{C}$, et al. Outcome and impact of surgery in paradoxical low-flow, low-gradient severe aortic stenosis and preserved left ventricular ejection fraction: A cardiac catheterization study. Circulation 2013; 128: S235-S242.

13. Nishimura RA, Otto CM, Bonow RO, Carabello BA, Erwin JP 3rd, Guyton RA, et al. 2014 AHA/ACC Guideline for the Management of Patients With Valvular Heart Disease: A report of the American College of Cardiology/American Heart Association Task Force on Practice Guidelines. Circulation 2014; 129: e521-e643.

14. Vahanian A, Alfieri O, Andreotti F, Antunes MJ, Baron-Esquivias $\mathrm{G}$, Baumgartner $\mathrm{H}$, et al. Guidelines on the management of valvular heart disease (version 2012). Eur Heart J 2012; 33: 2451-2496.

15. Lee SY, Shim CY, Hong GR, Seo J, Cho L, Cho IJ, et al. Association of aortic phenotypes and mechanical function with left ventricular diastolic function in subjects with normally functioning bicuspid aortic valves and comparison to subjects with tricuspid aortic valves. Am J Cardiol 2015; 116: 1547-1554.

16. Lee SY, Shim CY, Kim D, Cho I, Hong GR, Ha JW, et al. Factors determining aortic valve dysfunction in Korean subjects with a bicuspid aortic valve. Am J Cardiol 2017; 119: 2049-2055.

17. Sievers HH, Schmidtke C. A classification system for the bicuspid aortic valve from 304 surgical specimens. $J$ Thorac Cardiovasc Surg 2007; 133: 1226-1233.

18. Roberts WC, Ko JM. Frequency by decades of unicuspid, bicuspid, and tricuspid aortic valves in adults having isolated aortic valve replacement for aortic stenosis, with or without associated aortic regurgitation. Circulation 2005; 111: 920-925.
19. Lang RM, Bierig M, Devereux RB, Flachskampf FA, Foster E, Pellikka PA, et al. Recommendations for chamber quantification: A report from the American Society of Echocardiography's Guidelines and Standards Committee and the Chamber Quantification Writing Group, developed in conjunction with the European Association of Echocardiography, a branch of the European Society of Cardiology. J Am Soc Echocardiogr 2005; 18: $1440-1463$.

20. Briand M, Dumesnil JG, Kadem L, Tongue AG, Rieu R, Garcia $\mathrm{D}$, et al. Reduced systemic arterial compliance impacts significantly on left ventricular afterload and function in aortic stenosis: Implications for diagnosis and treatment. J Am Coll Cardiol 2005; 46: $291-298$.

21. Lang RM, Badano LP, Mor-Avi V, Afilalo J, Armstrong A, Ernande L, et al. Recommendations for cardiac chamber quantification by echocardiography in adults: An update from the American Society of Echocardiography and the European Association of Cardiovascular Imaging. J Am Soc Echocardiogr 2015; 28: $1-39 . e 14$.

22. Hachicha Z, Dumesnil JG, Pibarot P. Usefulness of the valvuloarterial impedance to predict adverse outcome in asymptomatic aortic stenosis. J Am Coll Cardiol 2009; 54: 1003-1011.

23. Halpern EJ, Mallya R, Sewell M, Shulman M, Zwas DR. Differences in aortic valve area measured with ct planimetry and echocardiography (continuity equation) are related to divergent estimates of left ventricular outflow tract area. Am J Roentgenol 2009; 192: 1668-1673.

24. Clavel MA, Malouf J, Messika-Zeitoun D, Araoz PA, Michelena HI, Enriquez-Sarano M. Aortic valve area calculation in aortic stenosis by CT and Doppler echocardiography. JACC Cardiovasc Imaging 2015; 8: 248-257.

25. Messika-Zeitoun D, Aubry MC, Detaint D, Bielak LF, Peyser PA, Sheedy PF, et al. Evaluation and clinical implications of aortic valve calcification measured by electron-beam computed tomography. Circulation 2004; 110: 356-362.

26. Eleid MF, Sorajja P, Michelena HI, Malouf JF, Scott CG, Pellikka $\mathrm{PA}$. Flow-gradient patterns in severe aortic stenosis with preserved ejection fraction: Clinical characteristics and predictors of survival. Circulation 2013; 128: 1781-1789.

27. Clavel MA, Dumesnil JG, Mathieu P, Pibarot P. Paradoxical low flow and/or low gradient severe aortic stenosis despite preserved LV ejection fraction: Impact of aortic valve replacement on survival. Eur Heart J 2010; 31: 954-954.

28. Pibarot P, Dumesnil JG, Bogaty P, Hachicha Z. Response to letter regarding article, "Paradoxical low-flow, low-gradient severe aortic stenosis despite preserved ejection fraction is associated with higher afterload and reduced survival". Circulation 2007; 116: E574-E574

29. Clavel MA, Cote N, Mathieu P, Dumesnil JG, Audet A, Pepin A, et al. Paradoxical low-flow, low-gradient aortic stenosis despite preserved left ventricular ejection fraction: New insights from weights of operatively excised aortic valves. Eur Heart $J$ 2014; 35: 2655-2662.

30. Chandra S, Rajamannan NM, Sucosky P. Computational assessment of bicuspid aortic valve wall-shear stress: Implications for calcific aortic valve disease. Biomech Model Mechanobiol 2012; 11: $1085-1096$

31. VanAuker MD, Chandra M, Shirani J, Strom JA. Jet eccentricity: A misleading source of agreement between Doppler/catheter pressure gradients in aortic stenosis. J Am Soc Echocardiogr 2001; 14: $853-862$.

32. Dyverfeldt P, Kvitting JP, Sigfridsson A, Engvall J, Bolger AF, Ebbers T. Assessment of fluctuating velocities in disturbed cardiovascular blood flow: In vivo feasibility of generalized phasecontrast MRI. J Magn Reson Imaging 2008; 28: 655-663.

33. Binter C, Gotschy A, Sundermann SH, Frank M, Tanner FC, Luscher TF, et al. Turbulent kinetic energy assessed by multipoint 4-dimensional flow magnetic resonance imaging provides additional information relative to echocardiography for the determination of aortic stenosis severity. Circ Cardiovasc Imaging 2017; 10: e005486.

\section{Supplementary Files}

Please find supplementary file(s);

http://dx.doi.org/10.1253/circj.CJ-19-0702 